\title{
E-Vote: Online Voting System for Campus Election
}

\author{
${ }^{1}$ Meera Asok, ${ }^{2}$ Nikhila Pillai, ${ }^{3}$ Sneha $S$ Chand, ${ }^{4}$ Vidya A, ${ }^{5}$ Smitha Vas P \\ ${ }^{1,2,3,4}$ B.Tech Student, ${ }^{5}$ Assistant Professor LBS Institute of Technology for Women, Trivandrum, \\ India. ${ }^{1}$ meeraanair8@gmail.com, ${ }^{2}$ pillai.nikhila@gmail.com, ${ }^{3}$ snehaschand@gmail.com, \\ 4vidyaa1998@gmail.com, 5smithavas2011@gmail.com
}

\begin{abstract}
E-voting means the voting process in elections by using electronic devices. E-voting promises the possibility of a convenient, easy and safe way to capture and count the votes in an election. This project provides the specification and requirements for e-voting using the CodeIgniter platform. The CodeIgniter platform is used to develop e-voting software. A voter may need to register for a particular election, and after successful registration an OTP will be generated and sent to the voter's registered mobile number or email id. If OTP entered is valid, the voter is directed to the login page. Else if OTP entered is invalid, voters will be instructed to re-enter OTP. In this work, a new e-voting system is proposed that ensures voter confidentiality and accuracy of voting, thereby providing an important framework based on unique identification, which is the admission number of the voter and his/her password used at the time of registration. The database of eligible voters is already kept in the software to avoid malpractice at the time of voter registration. Hence the software provides security at multiple levels. An online solution is very useful as the information about the voters and the election committee is also made available to the people in this system.
\end{abstract}

Keywords- AJAX, Apache, Bootstrap, CodeIgniter, Database, EVM, E-voting system, OTP.

\section{INTRODUCTION}

The most deliberate way in which each citizen can influence the government decision making is through voting. Voting is considered to be a formal procedure of expressing the preference for a candidate in a particular position .Campus elections are conducted to represent the student's union, space where students can share their thoughts, views, and ideas regarding college administration. This paper represents the idea of developing a website to conduct campus elections to minimize human effort.

E-vote uses electronic means to aid casting and counting of votes. Using internet support, a voter can cast their votes to corresponding candidates via pc or smartphone. This technique could make the entire procedure fast, simple, and quick both for admin and user. The proposed system includes levels of authentication techniques to ensure security;-user registration, one time password and voter login.

\section{RELATED WORKS}

\section{A. $\quad$ Paper Ballot System}

The voting method used in most of the campuses nowadays is through a paper ballot voting system. It operates by issuing paper ballots to eligible voters who present themselves at the polling unit on the day of the election. Here the voter is authenticated by searching for and ticking his or her name on the list of eligible students issued by the corresponding staff advisers. Then the voter has to collect the ballot paper containing the candidates list. Now the voter can cast their vote by ticking the column in front of their favorite candidate name. And then by folding that ballot paper, it is inserted into the ballot box. This is very time-consuming. It can be seen that no checksum or encryption is possible as data must be presented in clear text. In cases of high voter turnout, there is a high probability of queues formed by the voters. After the voting time ends, the casted votes through paper ballots are usually counted manually, which is a very time consuming process. Variation in result can occur due to any mistake in counting.

\section{B. Electronic Voting Machine (EVM)}

EVM is an electronic device used for recording votes. It came as a replacement to the paper ballot system. The standard means of conducting elections in India that comprises a control unit and balloting unit. The two units are joined by a five-meter cable. The Control Unit is with the Presiding Officer or a Polling Officer and the Ballot Unit is placed inside the voting compartment. Instead of issuing a ballot paper, the Polling Officer-in charge of the Control Unit will press the Ballot Button. This will enable the voter to cast his/her vote by pressing the blue button on the Ballot Unit against the candidate and symbol of his/her choice. The controller used in Electronic Voting Machines has its operating program etched permanently in silicon at the time of manufacturing by the manufacturer. No one (including the manufacturer) can change the program once the controller is manufactured. An EVM can be used to 
record a maximum of 2,000 votes. If an EVM stops working, it can be replaced with a new one and votes recorded until that time are safe in the memory of the control unit. The control unit can store the result in its memory until the data is cleared or deleted.

\section{PROPOSED SYSTEM}

In our proposed system, we design a website for conducting e-voting in the campus election using CodeIgniter platform that enables students to vote using a smartphone or PC. Initially, the voters would have to provide their registration number to authenticate themselves and establish their password. This constraint is imposed to ensure that only the genuine person is allowed to vote in the elections. Our main focus is on the session OTP that is provided to the voter after the registration process. The OTP is nothing but a One-Time Password that is required to continue with theprocess of voting and this makes the system secure and free from misuse by another person. After successful registration, the voter sets a password. At the time of voter login, a voter needs to enter the password which is set up during the registration process. Admin sets up a poll time within which voters can access the voting interface. After authentication, the valid voters can cast their votes before poll time ends. After the poll time gets completed, the admin generates the election results which can be viewed by the voters. The advantages of the proposed system include:

1. Reduce human effort

2. Secure user interface

3. Reduces complexity

4. Saves time

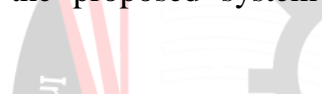

The new implemented voting protocol has 2 main modules:

\section{VOTER MODULE}

Figure 1 shows the flowchart of the voter module. The voter has the function of performing authentication and voting. The voter module allows users to register, login, cast vote, and logout. The user module supports user roles, which is set up with certain permissions allowing each to do only what the administrator permits. Initially, the student will have to undergo a registration process to become a voter. The admission number of the student must be entered during the registration process. If the admission number matches with the student database, then some credentials will be auto-filled. The student will have to give a password and a valid mobile number or email id for receiving an OTP as a part of verification. If the OTP entered is valid the student gets registered. To login to the voting interface, a registered student needs to authenticate by providing username and password. After the login session, the voter will be able to see the entire candidate's list and the profiles corresponding to each candidate. After selecting the desired candidates, the student will have to confirm the selected candidates and submit their choice. On completion, the student can log out of the voter module. After the election is completed, the winners will be automatically updated on the launch page for the voters to meet the new team.

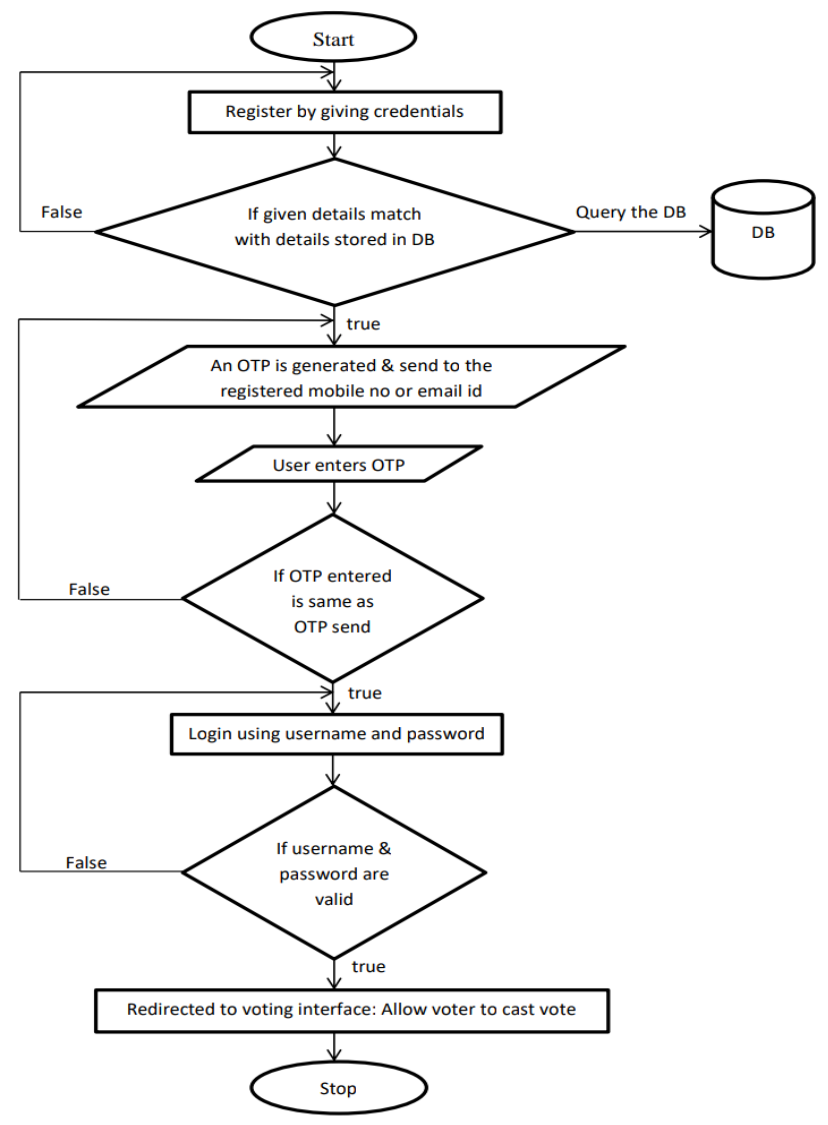

Figure 1. Flowchart of voter module

ADMIN MODULE

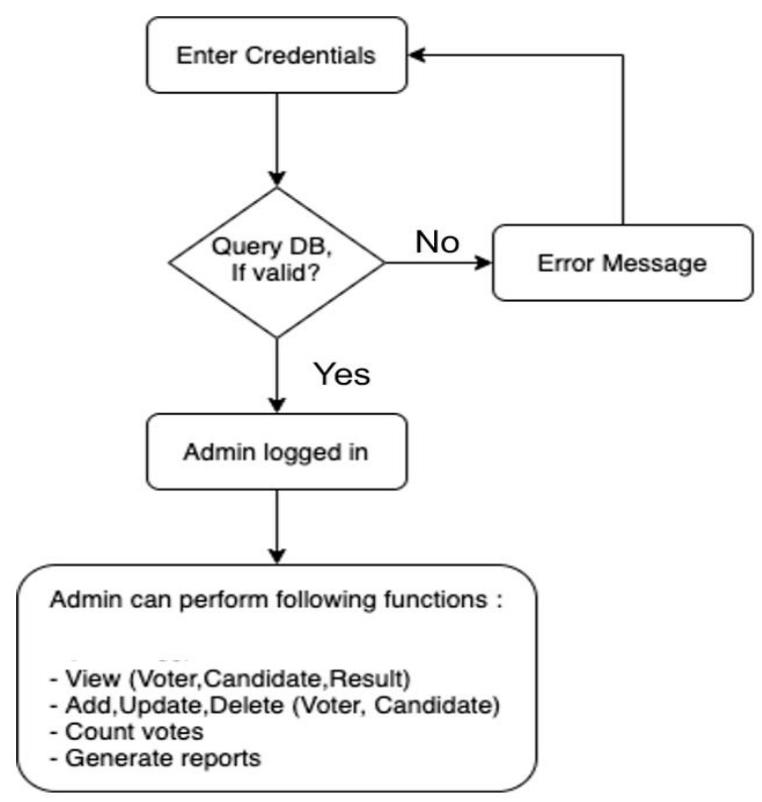

Figure 2. Flowchart of admin module

Figure 2 shows the flowchart of the admin module. The administrator can add, view and set permissions for the staff, add, view and edit voters, positions and candidates, 
can create a poll and generate the result. The admin module allows the system administrator to set up the back-end of the system and perform basic system configuration, the definition of predefined drop-down fields, definition of classes time schedule etc. Admin manages the voter module which provides users with definable access levels or roles. The admin login to the system using essential credentials and perform various roles for handling the website.

The admin roles include:

1. Admin can add new staff, view the details, set permissions, edit and delete it.

2. Admin can view the student list which can be sorted based on branch, year and status. He can also add, edit and delete the student list. The student list includes the list of all students in the campus

.3. Admin can view, sort and edit voters list. Voters list contains the list of students whose status is currently active, which implies, the students have already registered to the evote website.

4. Admin can add various positions, edit it and can also delete the entry. During this, the admin set permissions regarding the voters who can cast votes based on year and branch.

5. Admin is responsible for creating a new poll. For that, a poll name, start time, end time and positions competing in the election must be given.

6. Admin can add candidates who are going to compete in the election and selects the post to which the candidate competes in. Here the admin creates a candidate profile.

7. Finally in the result page, the admin can generate the result. Admin can view the result as either the whole result set which includes the details of all candidates who participated in the election along with the count of votes they received or as the winning team alone.

\section{ALGORITHM OF PROPOSED SYSTEM}

Step 1: Initialization of process.

Step 2: Student need to register by providing the details (Email ID, Phone Number)

Step 3: If the details given by the student matches with the existing student database, then an OTP (one time password) will be sent to the student's registered email-id / mobile number.

Step 4: If OTP entered is valid

a. Voter is directed to the login page.

b. Else if OTP entered is invalid, students will be instructed to re-enter OTP.

Step 5: After successful login, voter will be directed to voting interface

Step 6: When the poll time gets completed the admin filters the vote based on the designation of candidates, and directs to automatic result generation

\section{SYSTEM ARCHITECTURE}

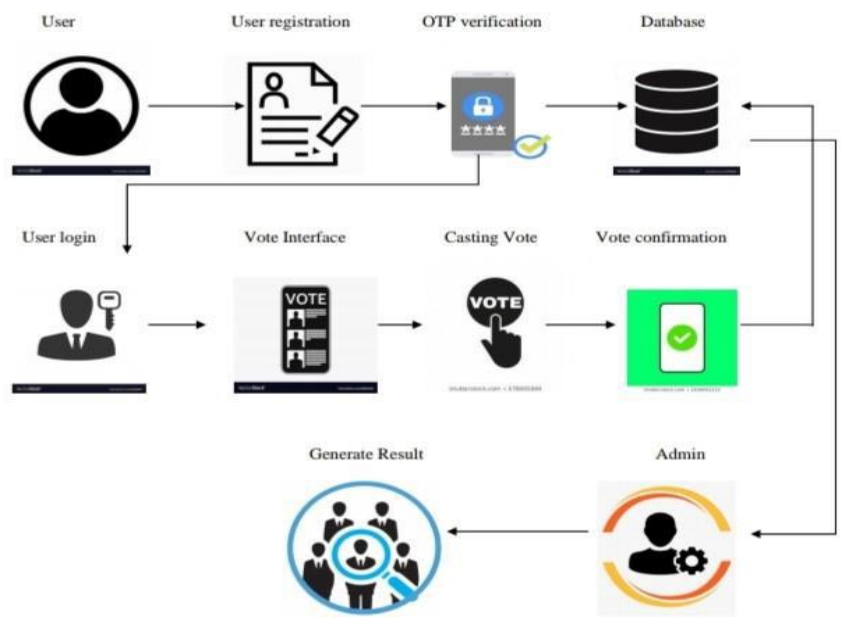

Figure 3. System Architecture

The overall architecture of the proposed system is shown in figure 3. Student's data is already stored in the DB server by admin. Students need to register by providing their details like admission number, email, phone number, and password via the website. If the entered details match with the details stored in the database, then an OTP will be sent to the student's registered mobile number or email id. Once the entered OTP is valid, students can $\log$ in to their account and vote online. A voter will be able to view the

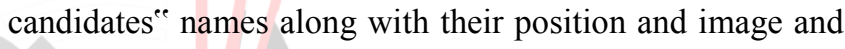
can cast a vote to the appropriate candidate. Similarly, the admin has to login using the credentials to access the admin section. In the admin section, the admin can add, edit, and sort the details of staff, students, voters, candidates, and their positions. Admin is responsible for setting the poll time within which the voter can access the voting interface. Once the poll time is completed the admin generates the result based on the designation of candidate and count of the vote. The admin can view the details ofthe total poll and the winning team. The voters will be able to see the winning team on the launch page itself.

\section{TECHNOLOGIES}

\section{A. CodeIgniter}

CodeIgniter is a PHP MVC framework that helps to speed up the development of web application. The framework uses the Model View Controller architectural design. It helps us to use models for data, controllers for control and views for HTML. MVC separates the data, business logic, and presentation. When a user requests a resource, the controller responds first. The controller understands the user request then requests the necessary data. It provides libraries for connecting to the database and performing various operations like sending emails, uploading files, managing sessions etc.

\section{B. Apache}

Apache is an open-source and free web server software used to serve web pages requested by client computers. 
Apache runs in the background under an OS, which supports multitasking, and provides services to other applications that connect to it. It can be installed on all operating systems like Linux, Windows, UNIX, Mac OS etc. It allows website owners to serve content on the web. When someone wants to visit a website, they enter a domain name into the address bar of their browser. Whenever it receives a request, it analyses the headers, applies the rules specified for it in the config file, and takes action. Then, the web server delivers the requested files by acting as a virtual delivery man.

\section{MySQL}

MySQL is the most popular Open Source Relational SQL Database Management System. MySQL is one of the best RDBMS being used for developing various web-based software applications. MySQL is developed, marketed and supported by MySQL AB. MySQL is very fast, reliable, and easy to use. The data in a MySQL database are stored in tables. It can be used to store anything from a single record of information to an entire list of student's data.

\section{NetBeans}

NetBeans is an IDE which allows applications to be developed from modules. Each module provides a welldefined function. It consists of an application platform that enables us to create web using PHP. It indents lines, matches words and brackets, and highlights source code syntactically and semantically. IDE is a free and open source integrated development environment for application development on Windows, macOS, Linux and Solaris operating systems. The IDE simplifies the development of web, enterprise, desktop and mobile applications that use the Java and HTML5 platforms. The IDE also offers support for the development of PHP and $\mathrm{C} / \mathrm{C}++$ applications. The IDE is licensed under the Apache License 2.0 .

\section{E. PhpMyadmin}

phpMyAdmin is a free and open source administration tool for MySQL that provides a convenient GUI for working with the MySQL database management system. As a portable web application written primarily in PHP, it has become one of the most popular MySQL administration tools, especially for web hosting services. Through this software you can create, alter, drop, delete, import and export MySQL DB tables. Typical operations such as the management of databases, tables, indexes, permissions are executed with the user interface. Basically, it is a thirdparty tool to manage the tables and data inside the database.

\section{E. AJAX}

Asynchronous JavaScript and XML is a technique for creating better, faster, and more interactive web applications with the help of XML, HTML, CSS, and Javascript. Ajax uses XHTML for content, CSS for presentation, along with Document Object Model and JavaScript for dynamic content display. With AJAX, when you hit submit, JavaScript will make a request to the server, interpret the results, and update the current screen. AJAX is a web browser technology independent of web server software. A user can continue to use the application while the client program requests information from the server in the background. A user can continue to use the application while the client program requests information from the server in the background.

\section{F. Bootstrap}

Bootstrap is a powerful toolkit - a collection of HTML, CSS, and JavaScript tools for creating and building web pages and web applications. It is a free and open source project, hosted on GitHub, and originally created by (and for) Twitter.Web designers and web developers like Bootstrap because it is flexible and easy to work with. Its main advantages are that it is responsive by design, it maintains wide browser compatibility, it offers consistent design by using reusable components, and it is very easy to use and quick to learn. With Bootstrap, web developers can concentrate on the development work, without worrying about design, and get a good looking website up and running quickly.

\section{PROGRAMMING LANGUAGES}

\section{Php}

Hypertext Preprocessor is open source software and a server-side scripting language used for creating dynamic web pages. It handles dynamic content, database as well as session tracking for the website. PHP as a web development option is secure, fast and reliable. PHP code is executed on the server and the result is returned to the browser as plain HTML. It can create, open, read, write, delete and close files on the server. PHP runs on various platforms including Windows, Linux, UNIX, Mac OS etc. It is compatible with almost all servers like Apache, IIS etc. It supports a wide range of databases and is integrated with a number of databases including MySQL, Oracle, and Microsoft SQL server.

\section{HTML}

Hypertext Markup language is a markup language used for creating web pages. Webpages are usually viewed in a web browser. It is used to create electronic documents that are displayed on the World Wide Web. Each page contains a series of connections to other pages called hyperlinks. It can be assisted by technologies such as cascading style sheets (CSS) and scripting languages such as JavaScript to form the frontend design of the website. It helps us to create a website or customize an existing web template. It is free of charge and no need to install any software. Web browsers receive HTML documents from a web server or from local storage and render the documents into multimedia web pages. HTML describes the structure of a web page semantically. HTML code ensures the proper formatting of text and images for the internet browser. Without HTML, a 
browser would not know how to display text as elements or load images.

\section{JavaScript}

JavaScript is a client side scripting language, which means the source code is processed by client's web browser. It is a programming language commonly used in web development, originally developed by Netscape as a means to add dynamic and interactive elements to a website. JavaScript enables interactive web pages and is an essential part of web applications. It is mostly used by all websites for the purpose of validation. In addition to validations, it supports external applications like PDF documents, running widgets, supporting flash applications etc. It can also load content into a document whenever the user requires it without even loading the entire page. JavaScript code can be inserted anywhere within the HTML of a webpage.

The JavaScript client-side mechanism provides many advantages like it allows you to check if the user has entered a valid e-mail address in a form field. It can be used to trap user-initiated events such as button clicks, link navigation, and other actions that the user initiates explicitly or implicitly.

\section{V1. RESULTS \& IMPLEMENTATION}
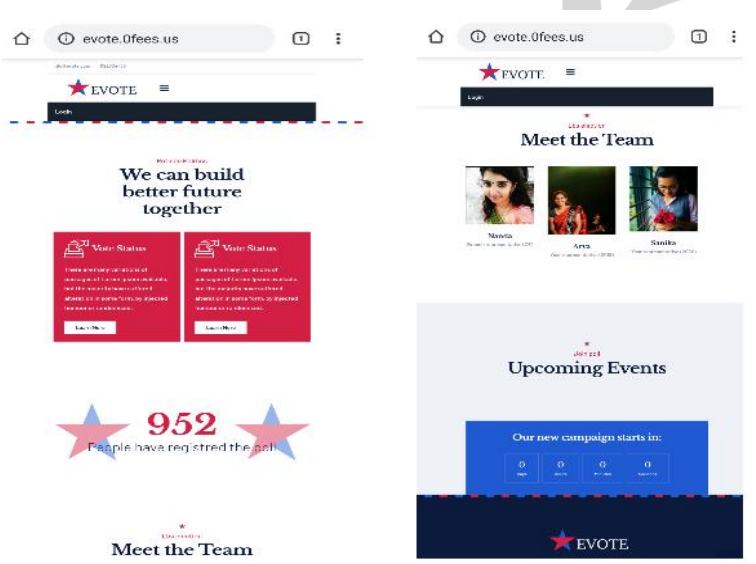

Figure 4. Launch page

Website: evote.Ofess.us-Figure 4 shows the Launch page. This is the main page of the E-vote website. Launch page contains a login section at the top part, displays the number of total registered users in the middle section \& after each election, the winning team will be displayed at the end of the page.
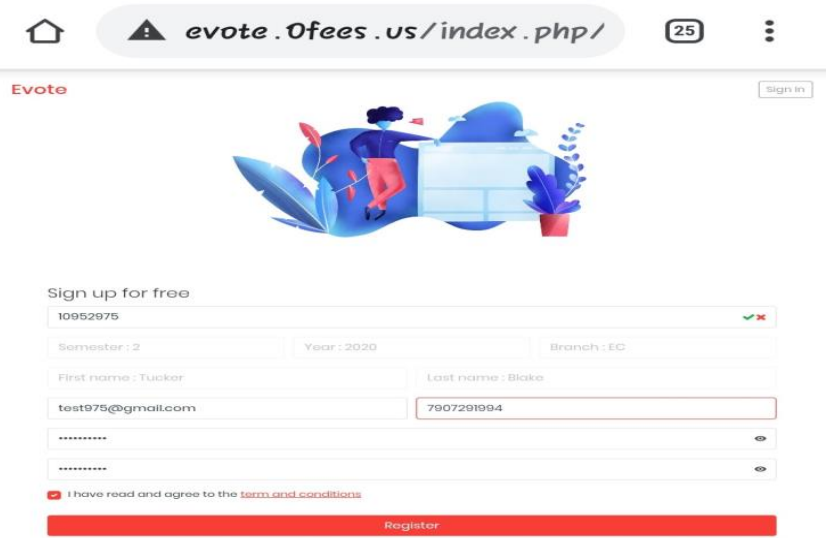

Figure 5. New User Registration
Figure 5 shows the user registration page. Any new user who enters the e-vote website must initially undergo a registration process where the user will have to fill details including admission number, mail id, mobile number and a new password to activate their account. Once the admission number is given the academic details of that number will be auto-filled. On giving email id and mobile number the backend performs a verification to confirm that no fraudulent activities happen. For that it performs a matching with a pre-existing student database. After confirmation, if the details match, the user is now able to register successfully.

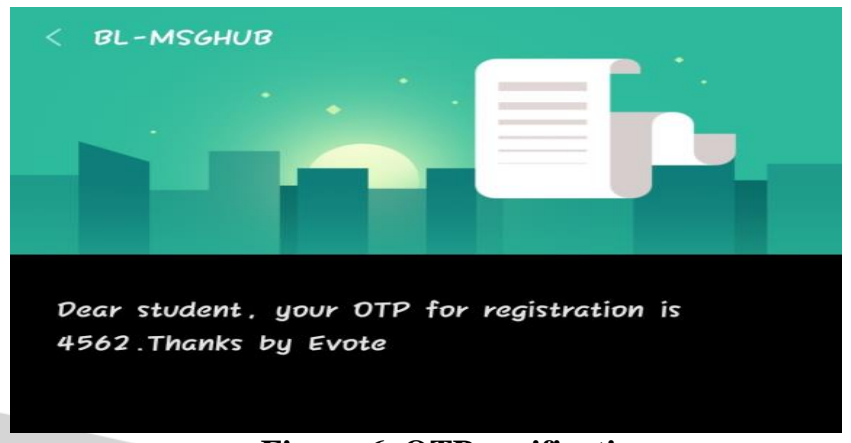

Figure 6. OTP verification

Figure 6 shows an OTP verification page. OTP sent to registered mobile/email-id.Once the registration is successfully completed, a One Time Password will be sent to the registered mobile number or email id. Users have to verify this in the OTP verification page of the website.

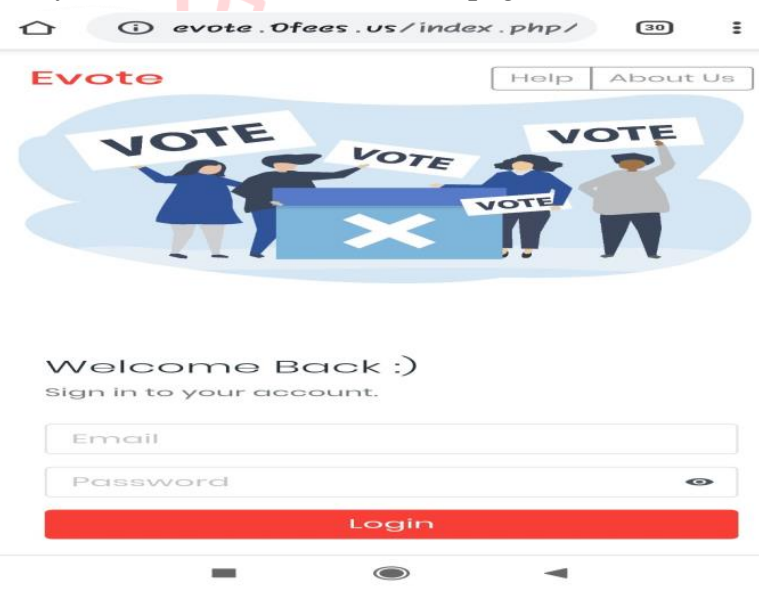

Figure 7. Login

Figure 7 shows the user login page. Once the OTP is verified the registered user is redirected to a Login page. Here the user will have to provide credentials like email and password, which was given during the registration process.

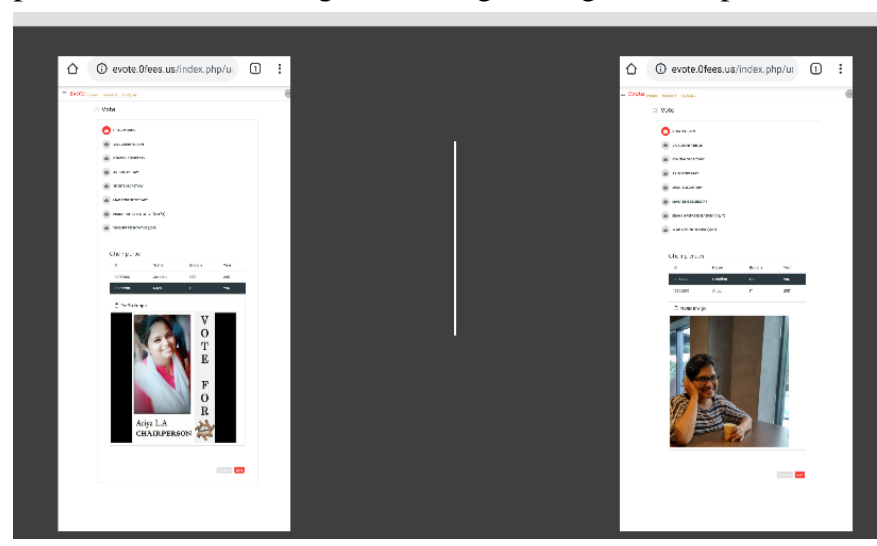

Figure 8. Voting interface 
Figure 8 shows the voting interface. After successful login, the voter enters a voting platform; the voter will be able to view each candidate with profile and can cast a vote.

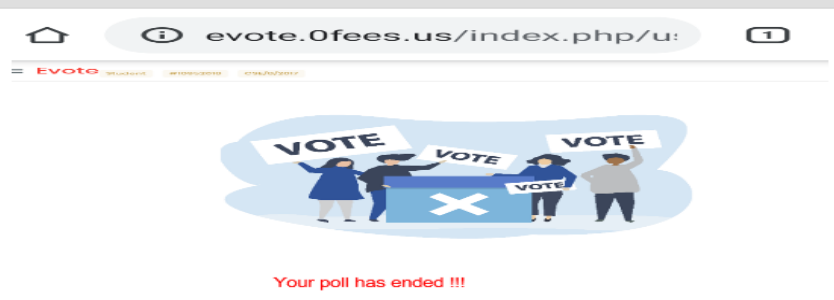

Figure 9. End page

Figure 9 shows the end page of the voting interface. If user tries to login after poll ends or login more than once, they are directed to the end page.

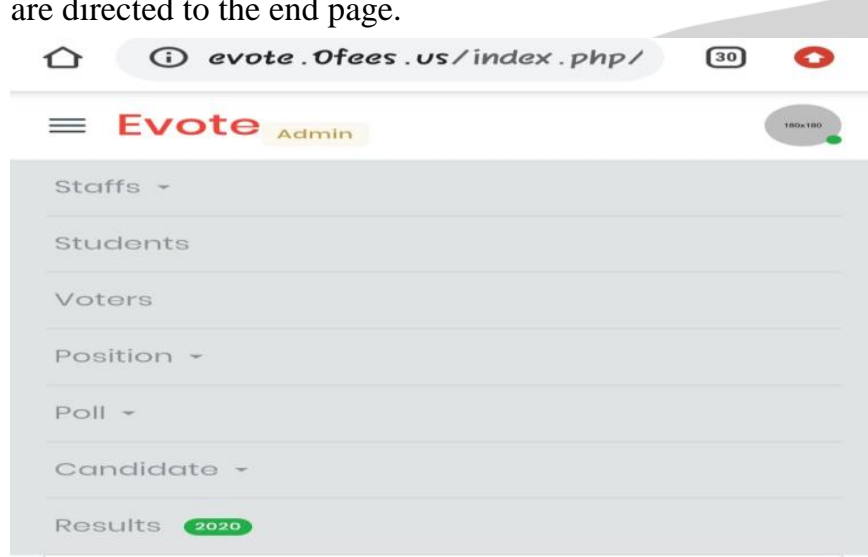

Figure 10. Admin interface

Figure 10 shows the Admin interface. Defines the permissions and roles associated with admin. The admin after logging into the system has entire control over the system. Admin can perform actions like Add, Delete, Update and View details regarding Staffs, Students, Votes, Positions, Poll and Candidate details and also can generate results.

\section{A evote. Ofees.us/index.php/ $25:$}

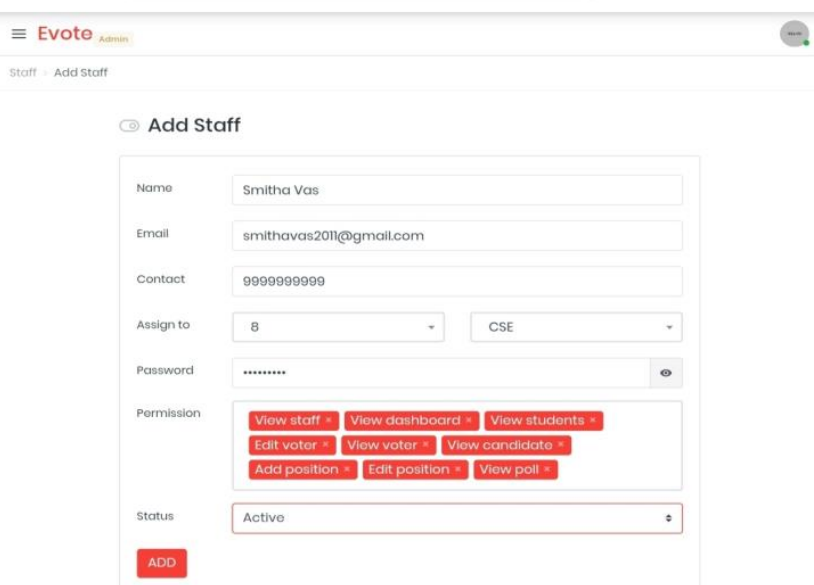

Figure 11 shows the admin role to add staff or sub admin using basic credentials, allocate branch and semester ,set password and permissions required.

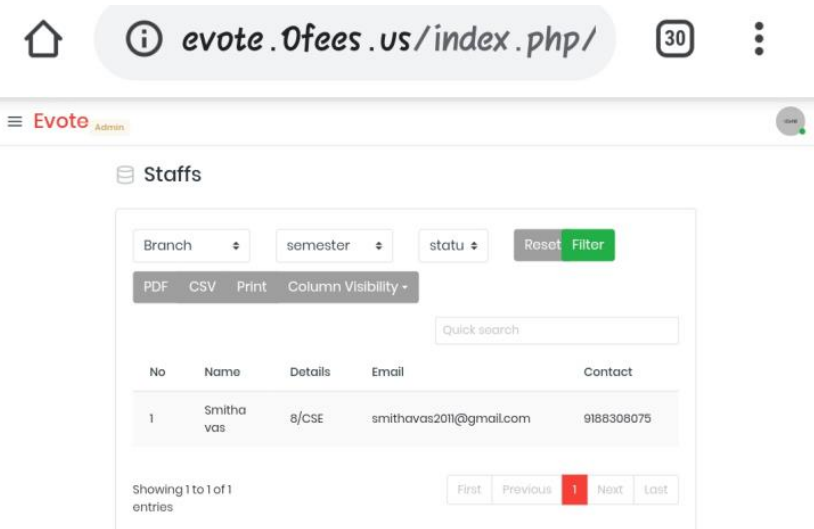

Figure 12. Edit/View Staff

Figure 12 shows the role of admin in view / edit staff details and filter thembased on branch, semester, and status.

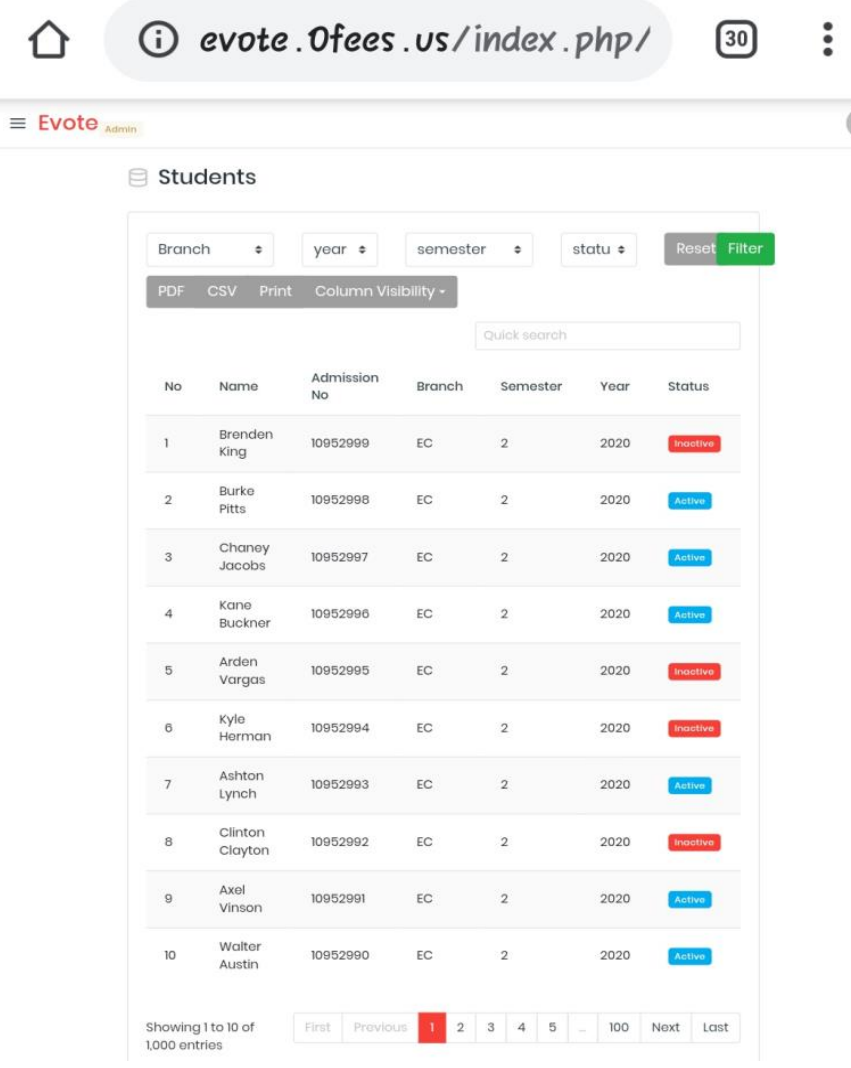

Figure 13. Student List

Figure 13 shows the List of all studentsin the college, both active and inactive. i.e. it contains the list of registered students and not registered students.

Figure 11. Add Staff 
$\equiv$ Evote

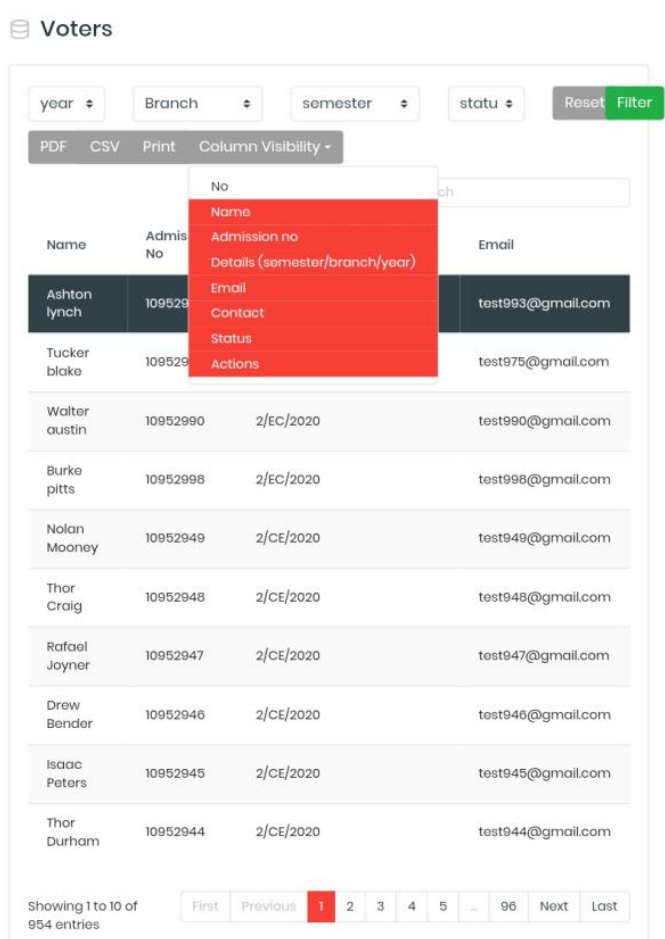

Figure 14. Voters List

Figure 14 shows the list of registered students. Registered students are the ones who complete the authentication processes including OTP verification.

$\equiv$ Evote

Position Add positon

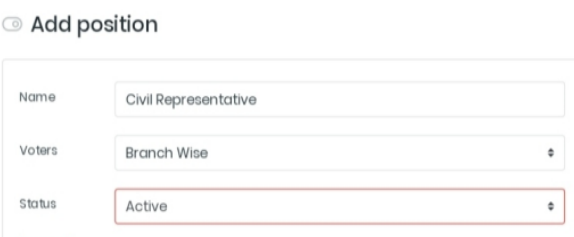

\section{Figure 15. Add position}

Figure 15 shows the role of admin to add candidate position, voters assigned and status. Voters assigned to each position depend on the branch and year of each voter. General posts require each voters to cast vote, but branch specific posts demand votes from respective branches.

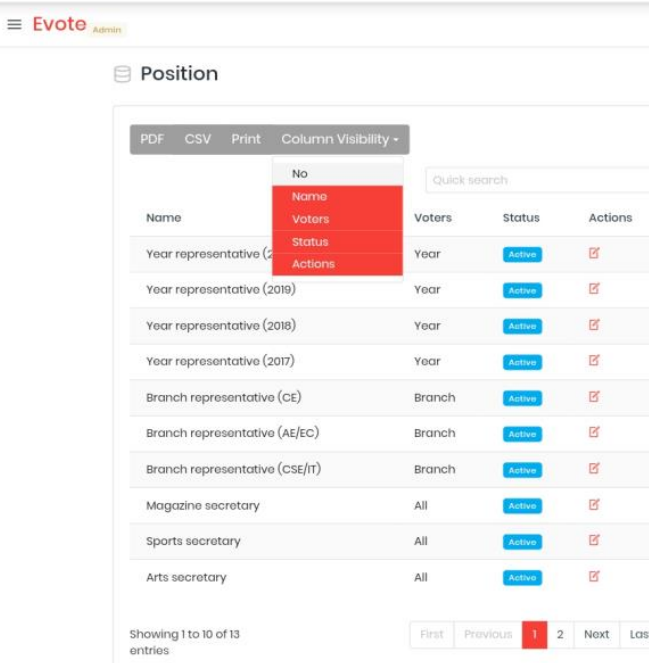

Figure 16. Edit/View Position

Figure 16 shows the role of admin to view/edit added candidate's position, voters assigned and status, i.e. whether the position is active or not during that poll.

$\equiv$ Evote

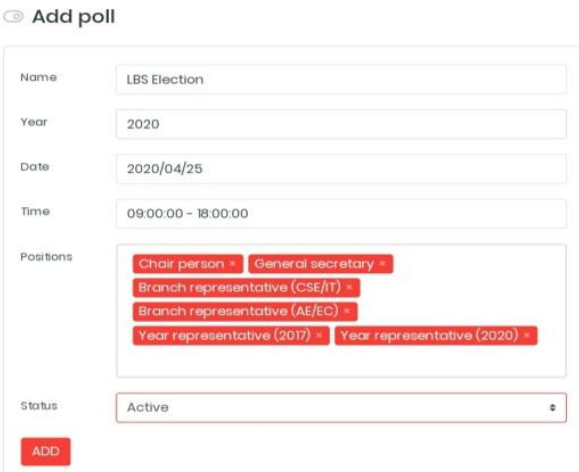

Figure 17. Add Poll

Figure 17 shows the role of the admin to add poll name, year and date of the poll, start time and end time of the poll and the positions that are competing in the current election.

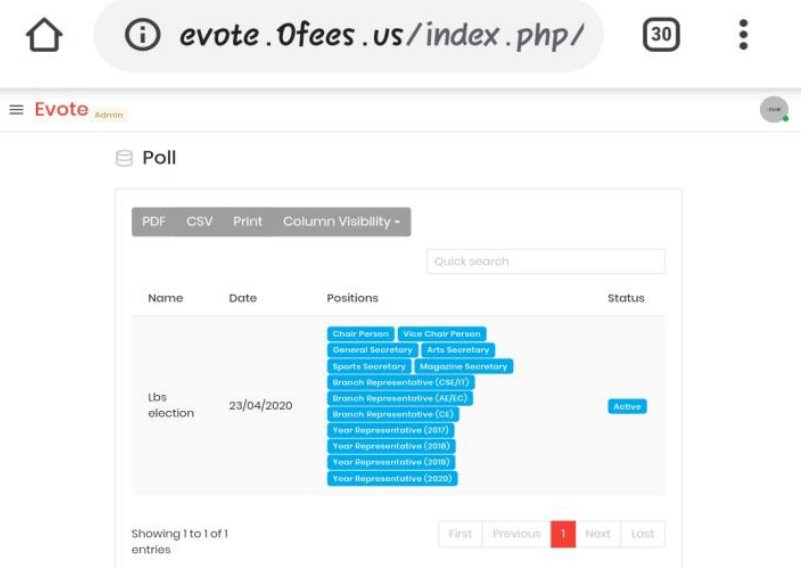

Figure 18. Edit/View Poll

Figure 18 shows the role of admin to view / edit poll details that the admin has added via the Add Poll. It contains details like the poll name. date of election, positions 
competing in the election, status (active/inactive) and action (edit/delete).

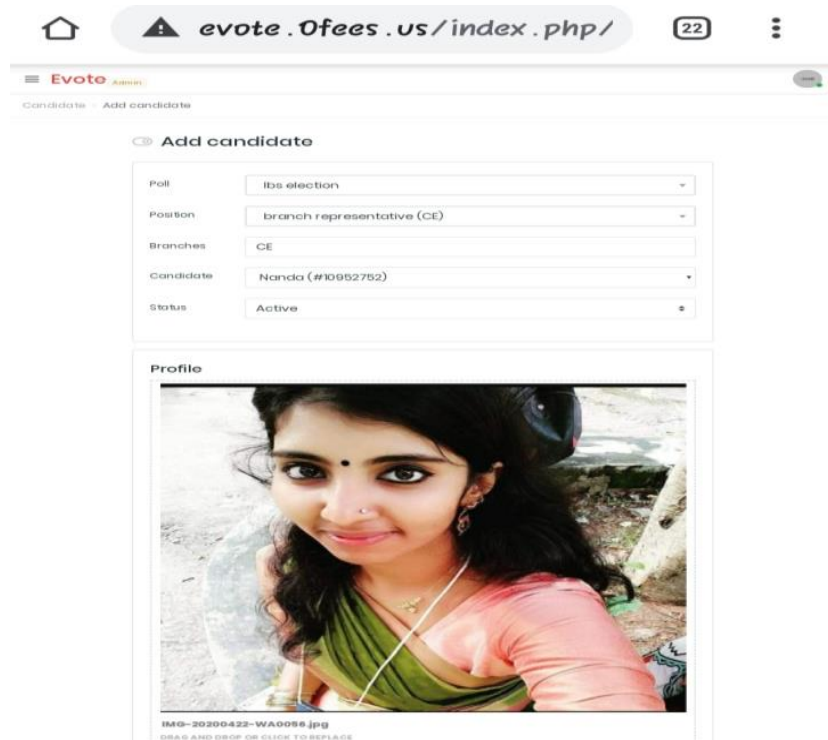

Figure 19. Add Candidate

Figure 19 shows the role of admin to add the candidate details. The required fields are poll name, position of the candidate, branch that can cast votes if it is branch specific, candidate name and the state of the position, i.e. whether active or inactive.

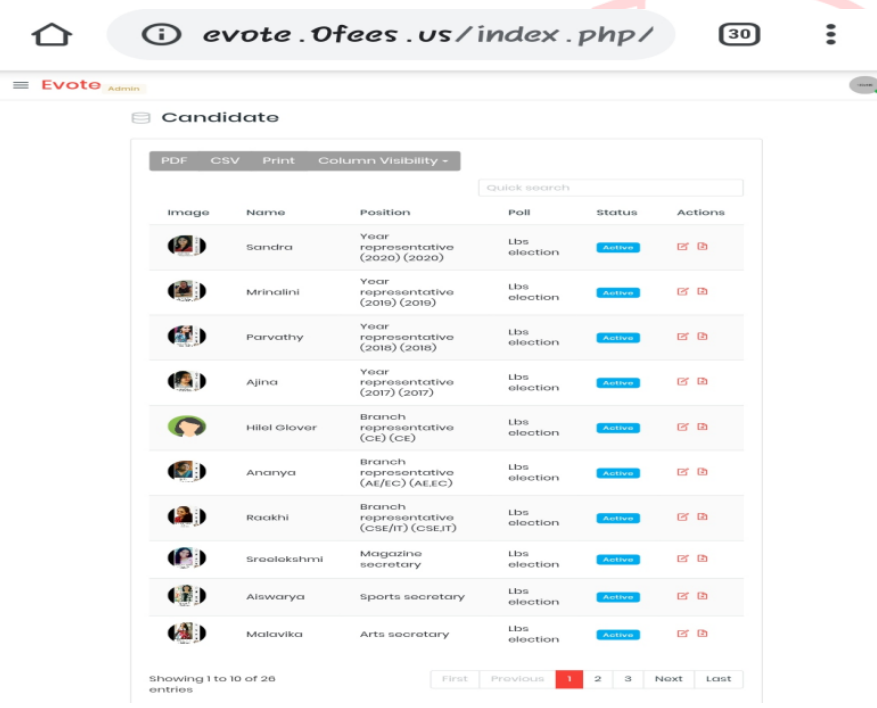

Figure 20. Edit/View Candidate

Figure 20 shows the role of admin to add/edit the candidate details that the admin entered in Add Candidate. It displays the profile of each candidate with picture, name, position, poll name, status (active/inactive) and action (edit/delete).

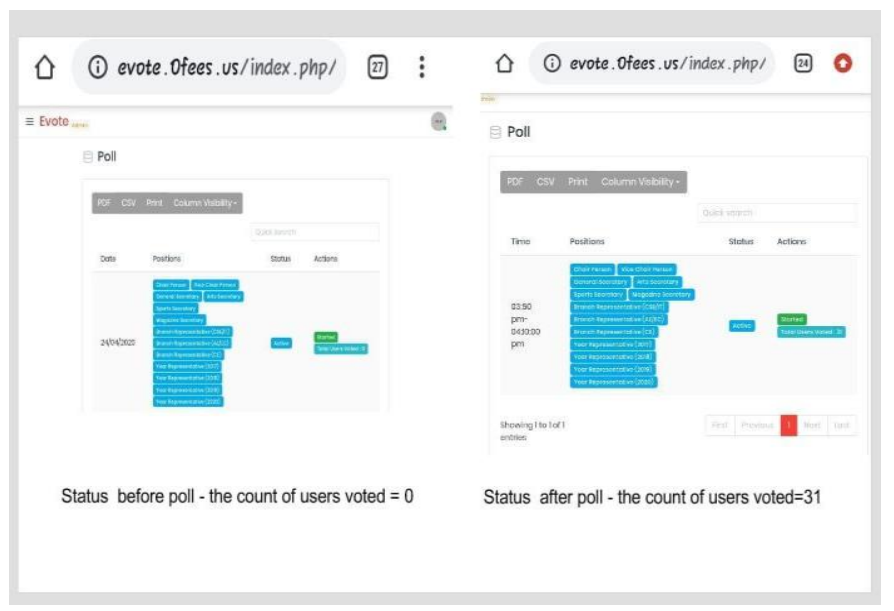

\section{Figure 21. Poll status}

Figure 21 shows the poll status i.e. the status before poll time and after poll time is completed. Initially the total number of users voted is 0 , but after the poll time is completed, i.e. after the election the number of poll gets incremented by the total users who voted in that election.

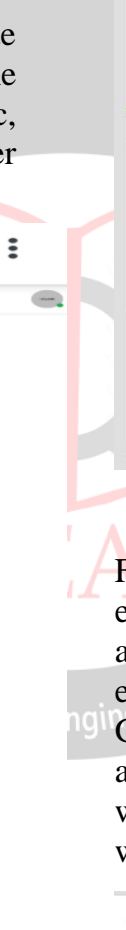

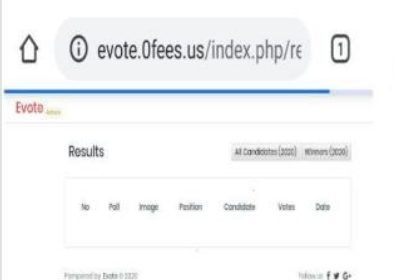

Results before poll
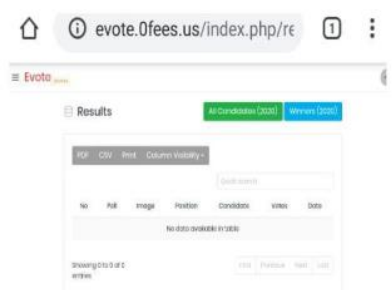

Results after poll with activated buttons to view al candidates list and winners list with corresponding votes.

\section{Figure 22. Result page}

Figure 12 shows how the admin could view the results after each election. i.e. the figure shows the results before poll and after poll. Initially the result page is empty. Once the election is completed the admin will have an option called Generate Result. The admin can then view all the results and he also has an option to sort the results to view the winners. Only after the admin generates the result, the voter will be able to view the winning team on the launch page.

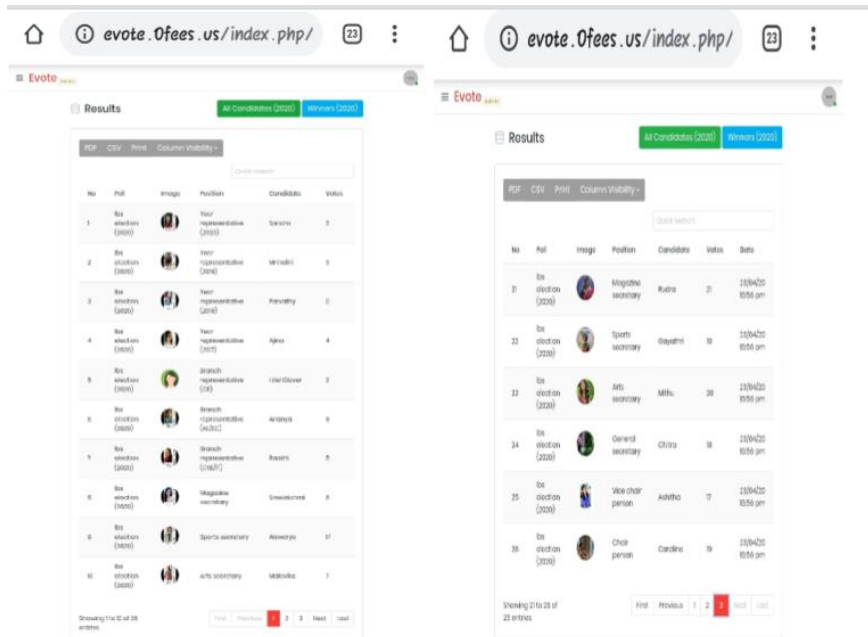

Figure 23. Candidate list with total votes 
Figure 23 shows the listof total votes obtained by each candidate in the election. The page contains poll name, image, position, candidate name and the number of votes corresponding to each candidate.

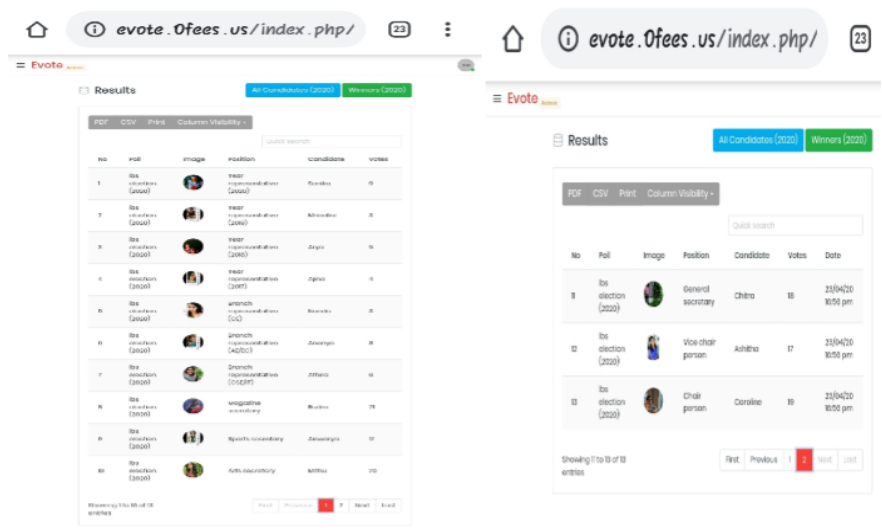

Figure 24. Winners List

Figure 24 shows the list of winners only. Admin has an option to sort the result to view the whole result of the election and the winners separately.

\section{V11. Conclusion}

We have proposed an e-voting system to conduct the online election in college campuses using user registration followed by user login and one-time password to the registered mobile or email id as an authentication technique. Thus security increases as there are 2 levels of authentication and it will overcome the problem of fake votes. This e-voting system would automate the current manual voting and focus on the online cast of votes by assuring safety and security. So basically there will be a database that will be maintained by the staff in which all the names of the voter with complete information are stored. This online voting system incorporates all the features of a regular voting system but offers an alternative method of conducting elections that is less stressful, easier, and faster through the use of a website. It is very difficult to design an ideal e-voting system that can allow security and privacy on a high level with no compromise. The practicable future scope of the project includes the improvement in the security level of the system by using a biometric authentication technique. Hence we suppose that this project will have greater scope and will provide a compact and stable system of voting. This system should be put to practice to phase out some of the problems we go through during manual voting. This system should be put to practice to phase out some of the problems we go through during manual voting. This proposed system is cost effective, reduces time consumption, automates manual process, increases the level of participation, moves parallel with the global environment of the online era and is user friendly.

\section{REFERENCES}

[1] PrashantPandit, SagarBhawar, Manisha Desai (2018)" Campus EVoting for Android and Web Based Application", International
Journal of Emerging Engineering Research and Technology, Volume 2 , Issue 7.

[2] Manivannan, K.Ramesh(2018), "E-VOTING SYSTEM USING ANDROID SMARTPHONE", International Research Journal of Engineering and Technology, Volume 2, Issue 06.

[3] AkinyemilAminatE (2018) "Biometrics Based E-Voting System" Computer Science Department Final Year Project, University of Ibadan.

[4] Akshay S. Agrawal, Mr.GuptaBhavesh, Maurya Ajay R, Mr.PhadnisHeramb B(2017), "OTP based e voting system", International Journal for Research in Engineering, Application \& Management, Vol-03, Issue 03.

[5] Preeti Ahlawat, Rainu Nandal (2016), "Performance Improvement using Pseudorandom One Time Password (OTP) in Online Voting System", IOSR Journal of Computer Engineering, Volume 17, Issue 5

[6] G.O.Ofori-Dwumfuo, E.Paatey (2015), "The Design of an Electronic Voting System", Research Journal of Information Technology, Volume:3

[7] Kashif H.M, Dileep Kumar and Syed Muhammad Usman (2017), "A Secure E-Voting System Based on Biometric Fingerprint Method", International Conference on Information and Intelligent Computing IPCSIT, Volume: 18

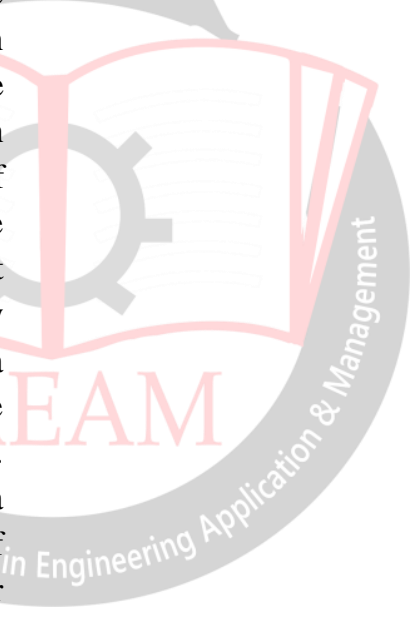

